\title{
SATELLITE OBSERVATIONS TO MONITOR SUBARCTIC RAIN-ON-SNOW EVENTS
}

\author{
Ludovic Brucker ${ }^{1,2}$, S. Joseph Munchak ${ }^{3}$ \\ ${ }^{1}$ NASA Goddard Space Flight Center, Cryospheric Sciences Lab., USA $;{ }^{2}$ Universities Space Research \\ Association, GESTAR, USA; ${ }^{3}$ NASA GSFC, Mesoscale Atmospheric Processes Lab., USA.
}

\begin{abstract}
Rain-on-snow (ROS) events have been the focus of numerous studies in the past five years. Their characteristics (frequency, extent, and duration) represent a new and relevant climate indicator. However, monitoring ROS occurrences remotely using satellite observations is deemed challenging. The ROS events can be sporadic, of very different intensities, and the outcome of the rain water uncertain (either it freezes in the snow cover or runs off). Using passive and active microwave remote sensing observations, our study proposes new approaches to monitor the occurrence of ROS events. Specifically, we utilize observations from Advanced Microwave Scanning Radiometer 2 (AMSR2), and Global Precipitation Measurements (GPM) Microwave Imager (GMI), and GPM Dual-frequency Precipitation Radar (DPR). We compare our ROS detection against weather stations and recently published algorithms using a different set of microwave frequencies.
\end{abstract}

Index Terms - precipitation, snow, rain on snow, passive microwave, radar

\section{INTRODUCTION}

Climate change in high northern latitudes is predicted to be greater in winter than in summer, and to have increasing, widespread impacts in northern ecosystems. Some of the resulting unknowns are the effects of an increasing frequency of sudden, short-lasting winter warming events, which can lead to rain on snow (ROS). Very little is known about ROS in northern regions, and even less about its cumulative impact on surface energy balance, permafrost, snow melt, and hydrological processes. Since, wintertime warming events have become more frequent in sub-Arctic regions, ROS event characteristics (frequency, extent, and duration) may represent new and relevant climate indicators. However, ROS event detection is challenging.

In this presentation, we propose new approaches to monitor the occurrence of ROS events using satellite passive and active microwave sensors. Specifically, we utilize observations from Advanced Microwave Scanning Radiometer 2 (AMSR2), Global Precipitation Measurements (GPM) Microwave Imager (GMI), and GPM Dual-frequency Precipitation Radar (DPR). GPM was launched in February, 2014. It operates multiple radiometers (in the frequency range
$10-183 \mathrm{GHz}$ ), and two radars ( $\mathrm{Ku}$ and $\mathrm{Ka}$ bands). GPM observations are used to quantify rainfall or snowfall rates and are thus appropriate to monitor ROS events up to $66^{\circ}$ North.

\section{METHOD OVERVIEW}

Recent studies [1-3] confirmed that the use of passive microwave observations is adequate to detect the occurrence of ROS events as originally investigated by Grenfell and Putkonen [4]. Using a larger range of frequencies (up to 89 $\mathrm{GHz}$ ), our satellite monitoring of the ROS event is based on both temporal and spectral variations in the satellite observations.

An empirical approach was chosen, using reference ROS events that occurred at different time of the year and in different regions (i.e. different environments taiga vs. tundra). The temporal evolutions and spectral signatures for all of AMSR2 channels, as well as all of the derived polarization and gradient ratios, were examined. The combinations that produced the clearest patterns corresponding to each phase of an ROS event (i.e. ROS liquid layer, ROS refreeze, post ROS re-freeze), and did so consistently for the three reference events were used. From these variables, three were selected for the temporal classification, and three for the spectral classification.

The relevance of the selected variables has been confirmed with snow radiative transfer simulation performed using DMRT-ML [5]. These modeling results will also be presented during this presentation.

\section{RESULTS}

First, our proposed algorithm is compared at a large scale to an implementation of [4]. In Figure 1, we notice that our use of both temporal and spectral detections reduces spurious detection (here on a given day in April) at a time when snow may melt due to warmer air temperature. Second, our ROS detection is compared locally to weather station observations (at Daring Lake, NWT, Canada). In Figure 2, we note that our algorithm has a timely detection of the first ROS event recorded by the station and that no false detection occurred due to melting snow (warm 2-m air temperatures). 


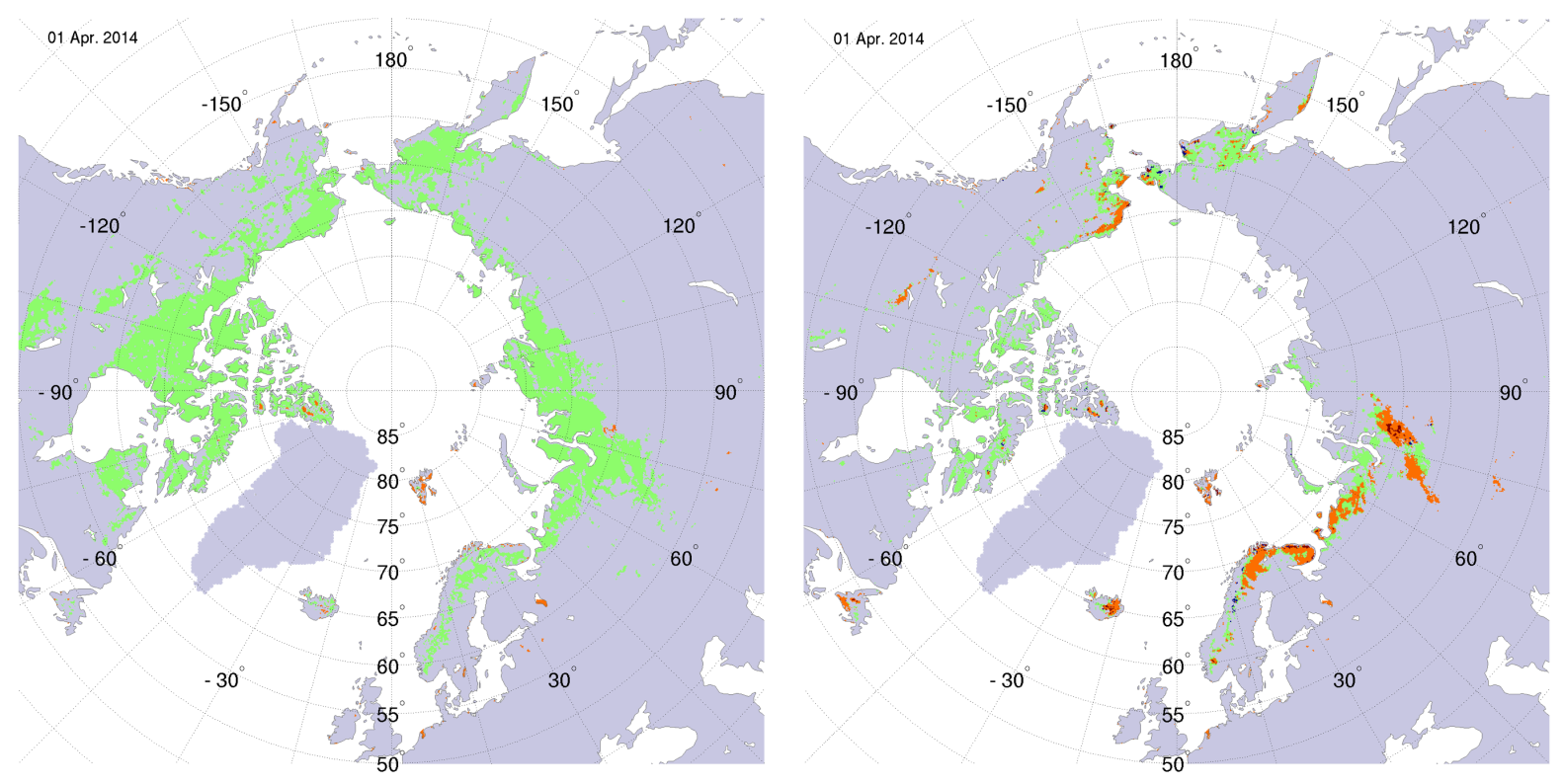

Figure 1. Rain-on-snow detection on 1 April 2014 using the method in [4] (left), and our algorithm based on both spectral and temporal detections (right). Our algorithm offers levels of confidence: red, orange, green indicates a decreasing level of confidence in our rain-on-snow detection.

In addition to the DMRT-ML modeling results, the ROS detection results are evaluated against atmospheric reanalysis from the European Centre for Medium-Range Weather Forecasts (ECMWF) ERA-Interim, and NASA Modern-Era Retrospective analysis for Research and Applications (MERRA). This initial evaluation in winter months shows that the proposed ROS detection using microwave sensors occur in areas that are shown by the reanalysis data to be experiencing liquid precipitation while there is snow on the ground.

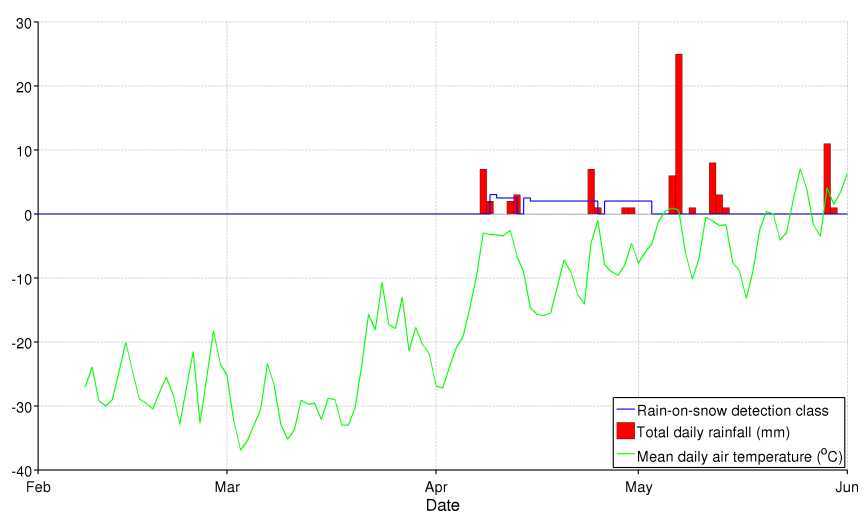

Figure 2. Rain-on-snow detection at Daring Lake, NWT (blue step line) and observations from a weather station (temperature (in ${ }^{\circ} \mathrm{C}$ ): green curve; and precipitation amount (in $\mathrm{mm}$ ): red bars).

\section{CONCLUSION}

This presentation will show the rain-on-snow detection results of our algorithm, which has been used recently in support of environmental studies [6]. We will also address the amazing potential of GPM active and passive observations for detecting on-going ROS events (Figure 3). This method is different since it only allows detection of on-going event during the satellite overpass.

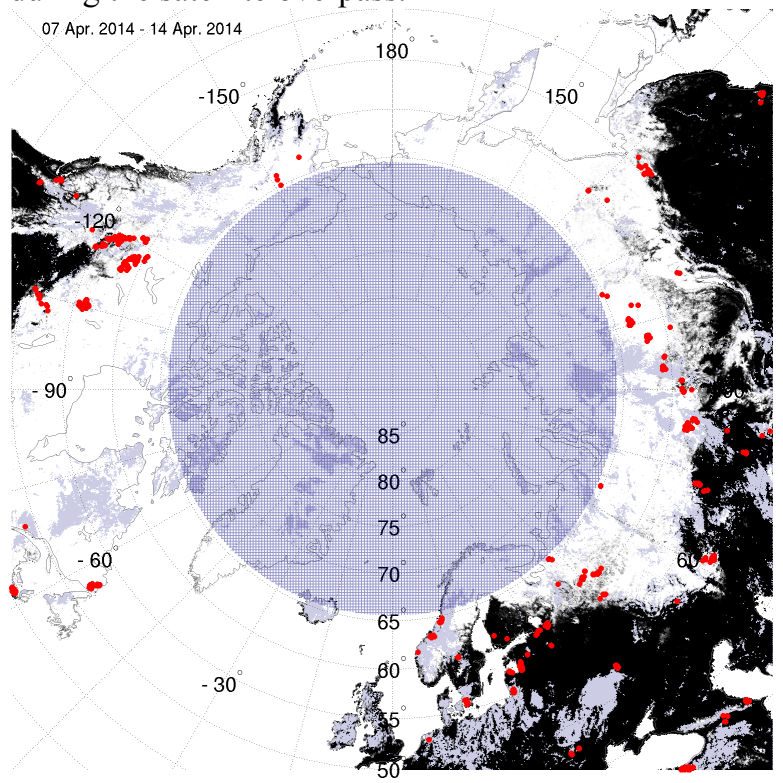

Figure 3. Rain-on-snow events during the week of 7-14 April 2014 detected by GPM (red dots), overlaid on the MODIS snow cover extent (white and grey). Region north of $66^{\circ}$ is masked out because not observed by GPM. 


\section{REFERENCES}

[1] Dolant, C., Langlois, A., Brucker, L., Royer, A., Roy, A., and Montpetit, B. Meteorological inventory of Rain-On-Snow events and detection assessment in the Canadian Arctic Archipelago using microwave radiometry. Physical Geography, in press, 2017, doi:10.1080/02723646.2017.1400339.

[2] Langlois, A., Johnson, C.-A., Montpetit, B., Royer, A., BlukaczRichards, E.A., Neave, E., Dolant, C., Roy, A., Arhonditsis, G., Kim, D.-K., Kaluskar, S., and Brucker, L. Detection of rain-on-snow (ROS) events and ice layer formation using passive microwave radiometry: A context for Peary caribou habitat in the Canadian Arctic. Remote Sensing of Environment Vol. 189, pp. 84-95, 2017, doi:10.1016/j.rse.2016.11.006.

[3] Dolant, C., Langlois, A., Montpetit, B., Brucker, L., Roy, A., and Royer, A. Development of a rain-on-snow detection algorithm using passive microwave radiometry. Hydrological Processes, 30, pp. 3184-3196, 2016, doi:10.1002/ hyp.10828.

[4] Grenfell, T. C., and J. Putkonen (2008), A method for the detection of the severe rain-on-snow event on Banks Island, October 2003, using passive microwave remote sensing, Water Resour. Res., 44, W03425, doi:10.1029/2007WR005929.

[5] Picard, G., Brucker, L., Roy, A., Dupont, F., Fily, M., Royer, A., and Harlow, C.: Simulation of the microwave emission of multilayered snowpacks using the Dense Media Radiative transfer theory: the DMRT-ML model, Geosci. Model Dev., 6, pp. 1061-1078, 2013, doi:10.5194/gmd-6-1061-2013.

[6] Sokolov, A., Sokolov, N., Ims, R., Brucker, L., and Ehrich, D. Emergent Rainy Winter Warm Spells May Promote Boreal Predator Expansion into the Arctic. Arctic, vol. 69, no. 2, pp. 121-129, 2016, doi:10.14430/arctic4559. 